\title{
Reflets
}

Revue ontaroise d'intervention sociale et communautaire

\section{Programme de promotion de la santé et du bien-être au travail : le travail par quarts... ça s’améliore}

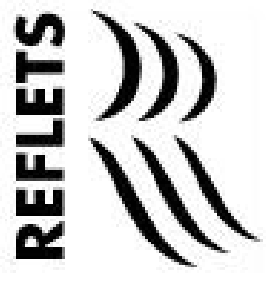

\section{Lise Barette, Louise Picard, Joanne Beyers et Teresa Taillefer}

Volume 1, numéro 2, automne 1995

La santé communautaire en Ontario français : défis et espoirs

URI : https://id.erudit.org/iderudit/026079ar

DOI : https://doi.org/10.7202/026079ar

Aller au sommaire du numéro

Éditeur(s)

Reflets : Revue ontaroise d'intervention sociale et communautaire

ISSN

1203-4576 (imprimé)

1712-8498 (numérique)

Découvrir la revue

Citer cet article

Barette, L., Picard, L., Beyers, J. \& Taillefer, T. (1995). Programme de promotion de la santé et du bien-être au travail : le travail par quarts... ça s'améliore.

Reflets, 1(2), 115-137. https://doi.org/10.7202/026079ar
Résumé de l'article

Le programme de santé et de bien-être au travail LE TRAVAIL PAR QUARTS... ÇA S'AMÉ-LIORE a été conçu par une équipe interdisciplinaire de Sudbury et Timmins afin de répondreà des besoins identifiés dans le nord de l'Ontario. Cet article discute des effets du travail parquarts et décrit le développement, la mise en oeuvre et l'évaluation d'un programme de promotionde la santé et du bien-être pour les travailleuses et travailleurs de quarts.
Tous droits réservés @ Reflets : Revue ontaroise d'intervention sociale et communautaire, 1995
Ce document est protégé par la loi sur le droit d'auteur. L'utilisation des services d'Érudit (y compris la reproduction) est assujettie à sa politique d'utilisation que vous pouvez consulter en ligne.

https://apropos.erudit.org/fr/usagers/politique-dutilisation/ 
Programme de promotion de la santé et du bien-être au travail:

\section{le travail par quarts... ça s'améliore}

Le programme de santé et de bien-être au travail LE TRAVAIL PAR QUARTS... ÇA S'AMÉLIO RE a été conç par une équipe interdisaplinaire de Sudbury et T immins afin de répondre à des besoins identifiés dans le nord de l' 0 ntario. C et artide discute des effets du travail par quarts et décrit le développement, la mise en oeuvre et l'évaluation d'un programme de promotion de la santé et du bien-être pour les travailleuses et travailleurs de quarts.

\section{Lise $B$ arette}

Infirmière en santé communautaire

Service de santé publique de Sudbury et du distric

\section{L ouise Picard}

Infimière en santé communautaire

Service de santé publique de Sudbury et du district

\section{Joanne B eyers}

$\mathrm{N}$ utritionniste de la santé publique

Service de santé publique de Sudbury et du district

\section{Teresa Taillefer}

D iététiste de la santé publique

Service de santé publique de Porapine

\section{Introduction}

Le travail par quarts existe depuis longtemps. C'est en fait grâce à l'invention de l'ampoule électrique par Thomas Edison qu'un 
«E nviron $25 \%$ de la main-d'oeuvre canadienne travaille par quarts... un tiers des F rancophones du $\mathrm{N}$ ord de l'O ntario travaillent par quarts. » plus grand nombre de gens ont pu travailler la nuit. Ce régime de travail continue à croître. Santé et B ien-être $C$ anada, dans son rapport L e travail par équipe, identifie plusieurs facteurs qui expliquent cette tendance (1992:1). N otons, entre autres, la nécessité d'assurer le rendement du capital investi par une production continue, la demande croissante pour des services accessibles 24 heures par jour et les récentes technologies en communication qui facilitent davantage le travail en dehors des heures traditionnelles de bureau.

Environ $25 \%$ de la main-d'oeuvre canadienne travaille par quarts ( $G$ roupe consultatif sur le temps du travail et la répartition du travail, 1994:16), ce qui représente près de trois millions d'individus selon Statistique $C$ anada. D'ici peu, une personne sur trois travaillera par quarts dans une variété de secteurs, soit le transport, la communication, les industries primaires et la fabrication, les services publics et services de santé et les services d'urgence. L'enquête sur la santé (ministère de la Santé de l'O ntario, 1990) indique que presque un tiers des Francophones du N ord de l'O ntario travaillent par quarts. U ne enquête effectuée auprès de 100 compagnies de Sudbury nous révèle que $43 \%$ des compagnies avaient établi des horaires par quarts et que, pour $23 \%$ de cescompagnies, au moins la moitié de leurs employés travaillaient par quarts (Service de santé publique de Sudbury, 1993). C es statistiques nous permettent de constater que, pour un nombre important de familles, le travail par quarts est une réalité qui fait partie intégrale de leur mode de vie.

Le présent article discute d'une recherche sur les effets du travail par quarts sur la santé et décrit le développement, la mise en oeuvre et l'évaluation d'un programme de promotion de la santé et du bien-être pour les travailleuses et travailleurs de quarts. Ce projet a été entrepris par les services de santé publique de Sudbury et de Porcupine et subventionné par la Direction de la promotion de la santé du ministère de la Santé de l'O ntario.

Pour quelles raisons les agences de santé communautaire devraient- elles s'intéresser à la question du travail par quarts? Tout d'abord, il importe de reconnaître les effets du travail par quarts et le lien avec la santé. 


\section{Les effets du travail par quarts}

L e travail par quarts se définit comme étant «un horaire de travail irrégulier dont les heures ne coïncident généralement pas avec I'horaire normal du travail de jour, soit de $7 \mathrm{~h}$ à $18 \mathrm{~h}$ » (M onk et Folkard, 1992:2). Ce terme englobe les quarts d'après-midi ou de nuit, les quarts par rotation, les quarts divisés et les heures de travail prolongées.

Figure 1

\section{Facteurs principaux sous-jacents à la tolérance au travail par quarts}

(adapté de Smolensky, 1980:62)

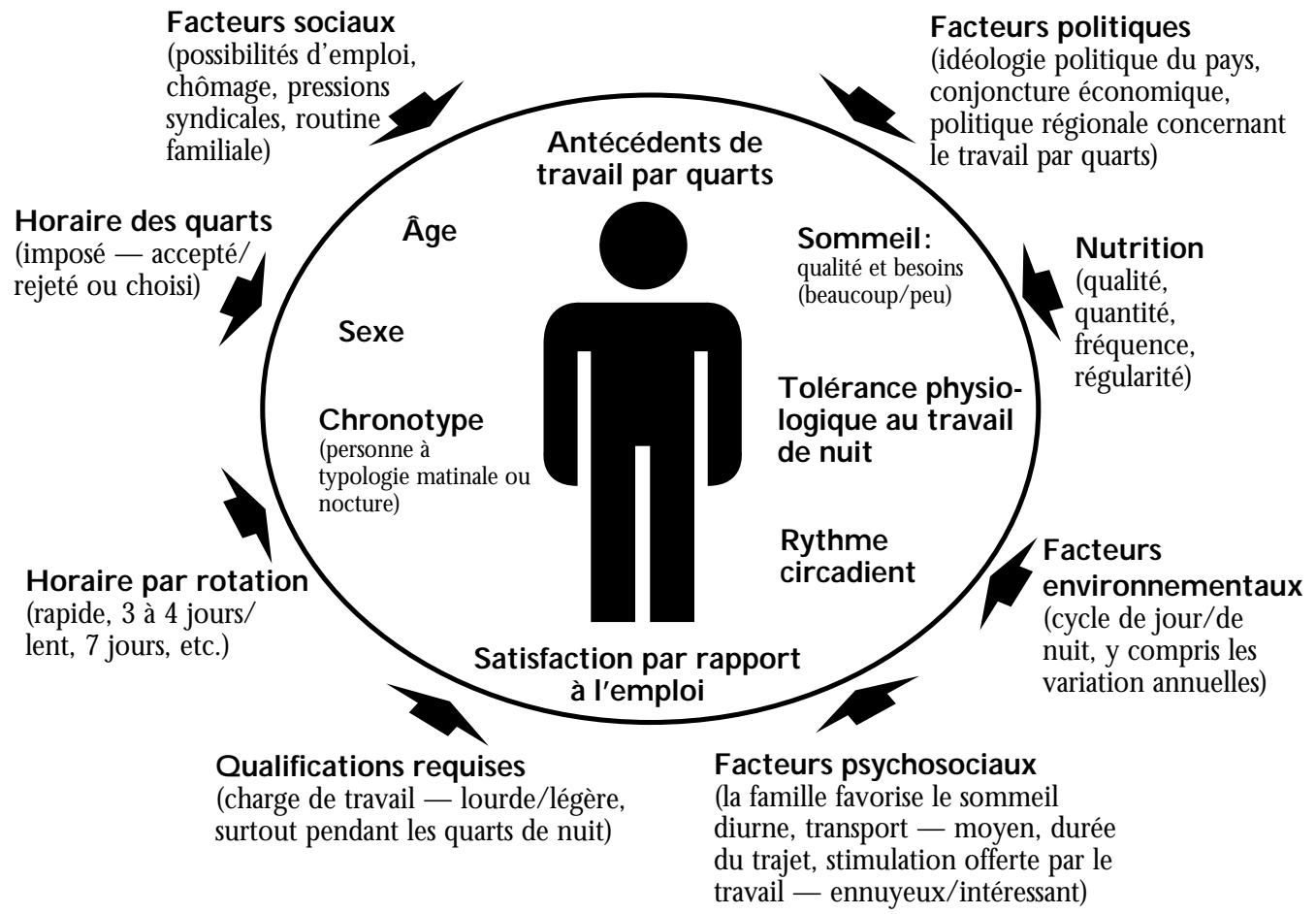


Plusieurs modèles ont été proposés pour expliquer les facteurs qui affectent la tolérance au travail par quarts (Santé et Bien-être social, 1992:8). Le modèle de Smolensky (1980:64) décrit les variables individuelles et externes qui s'unissent pour influencer la capacité d'une personne à s'adapter au travail par quarts. $N$ otre adaptation de ce modèle est présentée dans la Figure 1.

Cette figure représente bien les facteurs qui influencent le travail par quarts. II est toutefois essentiel de comprendre quels sont les effets de ce type d'organisation du travail sur la santé.

$C$ hacun d'entre nous est doté d'une horloge interne dont le rythme (de 25 heures) est lié à nos heures de sommeil, de loisir, de repos et de travail. Plusieurs études démontrent que cette horloge interne contrôle plusieurs fonctions biologiques telles que la température corporelle, le pouls, la tension artérielle, les fonctions rénales, la digestion, et les sécrétions hormonales. En général, ces fonctions sont plus intenses le jour et moins actives la nuit (C aldwell, 1995:19). II s'agit de notre cycle circadien qui, en fait, nous prédispose à travailler le jour et à dormir la nuit. Les changements dans la température corporelle illustrent bien ce principe (Figure 2). La température monte avant notre réveil et demeure élevée tout au long de la journée. C'est durant cette période que nous sommes plus vigilants, que notre digestion fonctionne mieux, etc. La température baisse ensuite durant la nuit, notre pouls et nos capacités de penser diminuant.

Le cycle circadien humain est en fait basé sur un horaire de 25 heures, mais nous réglons notre horloge à 24 heures grâce à des indices externes nommés z eitgebers (gardiens du temps) tels que la période de clarté et de noirceur et les routines journalières, comme par exemple les repas. II est donc facile de comprendre de quelle façon le travail par quarts peut dérégler notre cycle circadien. 
Figure 2

\section{Température du corps}

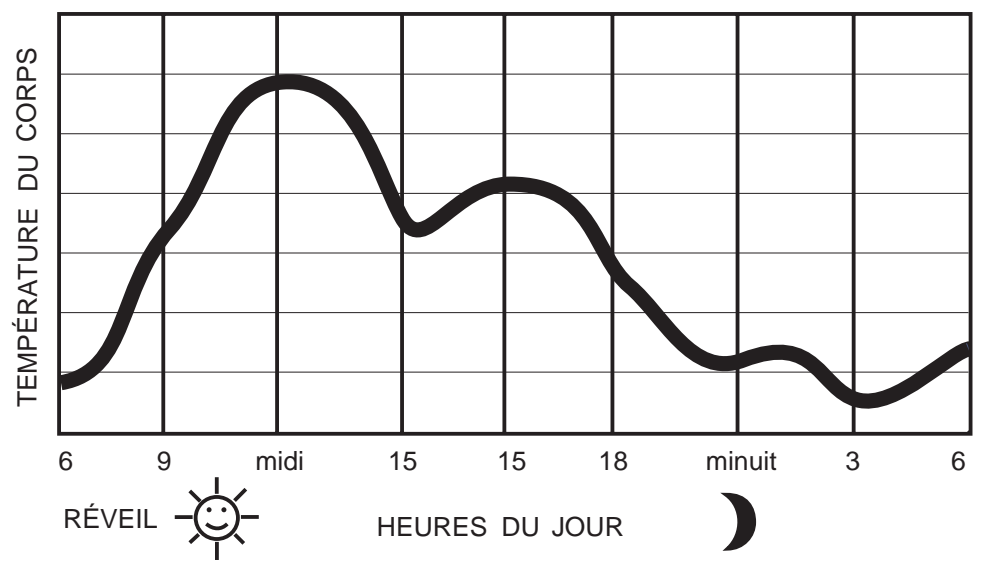

\section{Le sommeil}

«L es effets du travail par quarts incluent la modification des habitudes de sommeil et le manque chronique de sommeil.»
Les effets du travail par quarts incluent la modification des habitudes de sommeil et le manque chronique de sommeil. Près de 80 à $90 \%$ des travailleuses et travailleurs de quarts se plaignent de fatigue ou de somnolence au travail. (D otto,1990). II faut plusieurs jours avant que le corps ne s'ajuste à un changement dans son cycle de sommeil/ réveil. $D$ 'après certaines recherches, les rotations de travail rapides ou fréquentes et les rotations inverses semblent créer des difficultés d'adaptation particulières (M onk et Folkard, 1992:70). La fatigue qui en résulte est non seulement due à la pénurie de sommeil mais aussi à sa pauvre qualité. U ne nuit de sommeil se divise en quatre phases comme l'illustre la Figure 3. Le sommeil paradoxal (R E M sleep), qui se retrouve dans les deux dernières phases du sommeil, est indispensable pour la récupération mentale (C aldwell, 1995:56). Pour les travailleurs de nuit, le manque de sommeil paradoxal intensifie les effets liés au manque de sommeil. L'usage de somnifères et d'alcool peut également aggraver le problème. 


\section{Stades de sommeil}

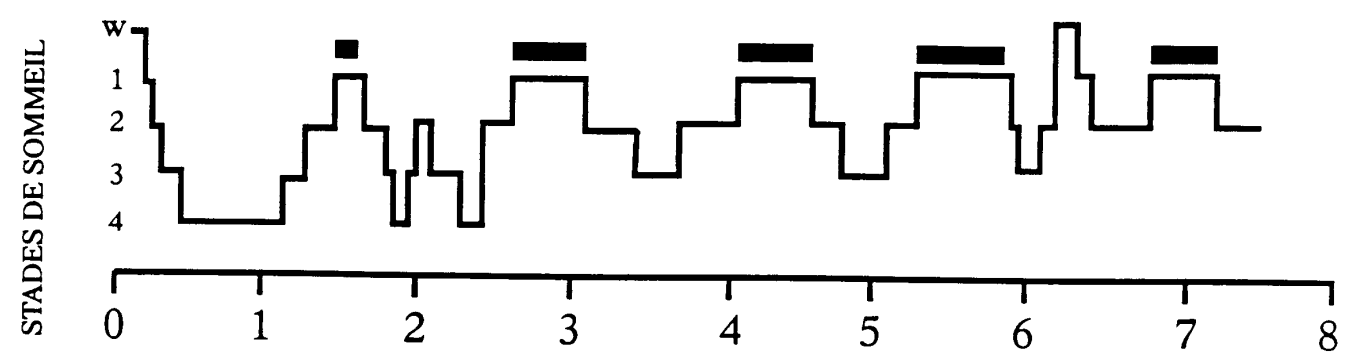

HEURES DE SOMMEIL

\section{La vie familiale et sociale}

L'une des plaintes les plus fréquentes des personnesqui travaillent par quarts concerne le bouleversement qui se produit dans leurs

«L es rôles de parent, de partenaire social et sexuel et de soignant sont tous affectés par le travail par quarts » relations sociales et familiales. Les rôles de parent, de partenaire social et sexuel et de soignant sont tous affectés par le travail par quarts (M onk et Folkard, 1990:21-22). Puisque leurs heures de sommeil, de travail et de loisir ne coïncident pas avec celles de la famille, il est difficile de partager ces expériences avec son partenaire ou avec ses enfants. Si nous ajoutons à cela les difficultés de conciliation des exigences familiales, professionnelles et sociales, il n'est pas surprenant de constater l'émergence de malaises psychosociaux et de tensions familiales. L'horaire de travail peut aussi contribuer à l'isolement social. Les études démontrent que les effets sont encore plusmarquéschez la femme qui travaille par quarts. Souvent, les attentes de la famille et de la société face à la mère demeurent les mêmes. De plus, les femmes requièrent généralement plus de sommeil que les hommes (M onk et Folkard, 1992:48).

Le stress chez les couples se manifeste par l'augmentation du taux de divorce pour les familles où l'un des partenaires travaille 
par quarts (Jung, 1986:163). Le fait que le couple passe moins de temps ensemble peut contribuer à un manque d'intimité et à des difficultés au niveau de leur communication et de leur vie sexuelle.

\section{Le stress}

Le stress créé par le travail par quarts a aussi des effets sur la capacité d'adaptation individuelle (M onk et Folkard, 1992). Plusieurs facteurs contribuent à cette capacité d'adaptation, notamment l'âge de l'individu, sa personnalité, ses antécédents médicaux, son sexe, sa forme physique et son attitude envers le travail par quarts (Santé et Bien-être C anada, 1992:12).

$D$ 'autres modèles suggèrent que le stress provient non seulement de l'horaire du travail par quarts mais aussi des tensions qui se développent chez l'individu qui essaie d'ajuster ses heures de sommeil et d'activité (C olquoun \& R utenfranz, 1980:ix). II ne faut pas oublier que la vaste majorité de gens qui travaillent par quarts ne le font pas par choix (Groupe consultatif sur le temps du travail et la répartition du travail, 1994:16)

\section{Mode de vie et nutrition}

Les recherches suggèrent que les personnes qui travaillent par quarts sont plus sujettes aux problèmes digestifs, à des problèmes de poids (Moore-Ede, 1993:3) et aux maladies cardiaques (K nutsson et al, 1986:89). C es problèmes sont reliés au mode de vie. En général, ceux et celles qui travaillent par quarts fument davantage, font moins d'exercices et consomment plus de glucides. Selon certaines études, la consommation de caféine, de drogue et d'alcool semble être plus élevée (Santé et Bien-être Canada, 1992:5). Toutefois, ces conclusions sont remises en question par certains auteurs (Wedderburn, 1993; Lenneras et al, 1993:248). S'il existe une association, qui n'est pas encore bien comprise, entre les modes de vie et le travail par quarts, aucun lien causal n'a par ailleurs été établi. 


\section{La sécurité et la vigilance au travail}

Pour les personnes qui travaillent par quarts, la fatigue est cause d'inquiétudes sérieuses pour la sécurité et la vigilance au travail. Le risque d'accidents augmente entre minuit et six heures ( $\mathrm{K}$ rieger, 1987). Plusieurs désastres industriels illustrent ce fait, dont ceux des usines nucléai res deT hree $\mathrm{M}$ ile Island, B hopal, $C$ hernobyl et celui de l'Exxon Valdez.

Le dérèglement du cycle circadien nous offre certaines explications. II faut se rappeler, par exemple, qu'entre 2 heures et 5 heures la température corporelle est à son niveau le plus bas. La mémoire à court terme, les processus cognitifs et la rapidité de réaction sont réduits. C ertaines personnes qui travaillent par quarts parlent de la paralysie du shift. C ette diminution de l'efficacité de la performance, de même que le risque d'accident, sont des effets qui préoccupent particulièrement les employeurs et les motivent à appuyer les programmes de promotion pour les employés

Étant donné les multiples effets du travail par quarts, il n'est donc pas surprenant que la question d'adaptation à ce travail ait été jugée importante.

\section{Limites de nos connaissances}

La recension des écrits suggère qu'en dépit du grand nombre de recherches sur le travail par quarts, il existe peu de réponses claires en ce qui a trait à la santé et la nutrition des travailleurs et travailleuses. C eci s'explique en partie par les problèmes méthodologiques associés aux recherches du travail par quarts (Wedderburn, 1993; Colligan et R osa, 1990). Il importe de reconnaitre les multiples configurations possibles du travail par quarts. II existe des centaines de différents horaires possibles et chaque genre d'horaire a ses propres avantages et désavantages. Le fait que la majorité des recherches se limitent aux hommes est un autre point important à considérer dans l'interprétation des résultats. 
De plus, dans toutes les recherches touchant la santé au travail, certains biais doivent être pris en considération afin d'éviter de fausses conclusions. Par exemple, «'effet du travailleur en bonne santé» (ETBS) est un phénomène qui joue sur ce genre de recherche. II s'agit du fait qu'en principe, les personnes ayant de la difficulté à s'adapter au travail par quarts (pour des raisons médicales, sociales ou autres) ont peut-être choisi de démissionner ou de travailler de jour. II se pourrait alorsque les personnesqui continuent à travailler par quarts soient des personnes dont la santé est particulièrement bonne L'ETBS peut donc constituer un gauchissement non intentionnel dont il faut tenir compte dans l'interprétation des études sur la santé au travail.

Les études disponiblesétablissent néanmoins un lien clair entre le travail par quarts et la santé, tout en soulignant le besoin de poursuivre la recherche afin de clarifier les contradictions qui y existent. C hose certaine pour les travailleuses et travailleurs par quarts, vivre sainement présente des défis particuliers qui font appel à la collaboration de tous ceux qui s'intéressent à la promotion de la santé et du bien-être au travail, y compris les services de santé publique.

Le programme de santé et de bien-être au travail LE TR AVAIL PAR QUARTS... ÇA S'AM ÉLIOR E a donc été élaboré afin de répondre à des objectifs spécifiques. Voici quelques-uns des facteurs ayant contribué à la mise en oeuvre de ce projet.

- Plusieurs industries du nord de l'O ntario telles que les industries forestières, minières et hydroélectriques, ainsi que les professionnels de la santé et sécurité au travail de diverses entreprises, étaient à la recherche d'aide pour aborder cette question. D eux cents employés d'une mine du nord de l'O ntario avaient participé à un projet pilote ayant permis d'identifier des besoins précis quant à l'adaptation au travail par quarts.

- U ne analyse bibliographique exhaustive a permis d'identifier des lacunes sérieuses au niveau de connaissances en matière de santé reliée au travail par quarts. Les ressources et les programmes éducatifs existants ne sont pas appropriés ou ne répondent pas aux besoins du milieu de travail (par exemple, le contenu, la clarté de la langue, les principes d'apprentissage 
non adaptés aux adultes ou une durée incompatible avec les contraintes de temps du milieu du travail).

- De plus en plus, les employeurs ont constaté que la promotion de la santé auprès des employés était une composante essentielle à un milieu de travail sain et à un meilleur rendement au travail.

\section{Description du programme}

Pendant I'hiver 1992-93, les nutritionnistes du nord-est de I'O ntario se sont penchés sur les questions suivantes. Existe-t-il une alimentation particulière qui facilitera l'adaptation au travail par quarts? Q uelles stratégies et ressources sont disponibles pour encourager une alimentation saine chez les travailleurset travailleuses de quarts?

En constatant la pénurie de recherche dans le domaine et I'intérêt des industries, des nutritionnistes et des infirmières hygiénistes de Sudbury et deT imminsont missur pied une équipe interdisciplinaire qui a décidé de se pencher sur une problématique plus globale, soit les défis en matière de santé et d'alimentation que doivent relever les travailleurs et travailleuses de quarts.

A près consultations auprès des intervenants clés, la D irection de la promotion de la santé et les partenaires de travail, les services de santé publique de Sudbury et de Porcupine ont élaboré le programme de santé et de bien-être au travail LE TRAVAIL PAR QUARTS... ÇA S'AMÉLIORE.

V isant à améliorer la santé des travailleurs et travailleuses de quarts de l'O ntario, ce programme comporte quatre objectifs:

- sensibiliser et renseigner davantage les employés et employeurs sur les effets du travail par quarts sur la santé, y compris l'alimentation, le sommeil, le mode de vie et la vie familiale et sociale, en développant des ressources appropriées,

- augmenter les connaissances au sujet des liens entre le travail par quarts et la santé, 
- établir et améliorer des partenariats nécessaires entre les employés, les employeurs, les syndicats et les pourvoyeurs de services afin de mieux promouvoir la santé des travailleuses et travailleurs par quarts.

- faciliter le dével oppement de politiques visant à créer un environnement sain et à favoriser un meilleur rendement au travail.

Ces objectifs se sont traduits en trois activités locales, concrètes et réal isables, soit le développement de ressources et de stratégies éducationnelles, l'établissement d'une banque de données sur I'impact du travail par quarts sur la santé du travailleur du nord de l'O ntario et l'établissement des partenariats dans les milieux de travail.

\section{Développement des ressources et stratégies éducationnelles}

\section{Guide des employés}

Le guide des employés propose des stratégies d'adaptation pour les travailleurs et les travailleuses de quarts. II offre un aperçu des effets de ce genre de travail et des stratégies d'adaptation et contient aussi une liste de ressources suggérées, des recettes et même des activités pour les enfants. Les stratégies qui y sont discutées touchent le sommeil, la vie familiale, l'activité physique et une saine alimentation.

\section{Guide de l'animateur}

Le guide de l'animateur ou de l'animatrice offre un outil à toute personne intéressée à la promotion de la santé des personnes qui travaillent par quarts. Les utilisateurs ciblés sont les infirmières et infirmiers hygiénistes et en santé du travail, les nutritionnistes en santé publique, le personnel en santé et sécurité du travail, les éducateurs et les éducatrices du domaine de la santé et les animateurs 
et animatrices communautaires qui ont un diplôme dans un domaine connexe à la santé. Jusqu'à présent, 80 participantset participantes du nord-est de l'O ntario ont reçu la formation d'animateur et d'animatrice pour ce programme.

C e guide est auto-dirigé et facile d'emploi; il suggère un horaire qui facilitera la planification du programme dans le milieu de travail et propose certaines activités et stratégies préparatoires soulignant l'importance d'obtenir l'appui des personnes clés du milieu du travail. D es suggestions sont offertes pour deux étapes importantes, soit l'analyse du milieu de travail et la maximisation des opportunités et des ressources. U ne dernière partie décrit un plan de promotion du programme et offre des ressources telles que des affiches et des dépliants promotionnels qui peuvent être distribués dans l'enveloppe de paie. U ne bibliographie détaillée complète le guide.

Ce guide a été conçu pour encourager les animatrices de permettre aux travailleuses et aux travailleurs de quarts de partager leur vécu et leurs stratégies d'adaptation avec leurs collègues de travail. Si l'animateur ou l'animatrice a déjà travaillé par quarts ou a vécu avec un partenaire travaillant par quarts, il sera en mesure de partager son expérience et aura alors une meilleure crédibilité.

\section{Atelier pour les employés}

La première partie de l'atelier consiste en une auto-évaluation de tous les participants et vise plusieurs buts: permettre de personnaliser le contenu de l'atelier selon les besoins identifiés par les participantes et les participants; déclencher une réflexion personnelle et stimuler la discussion; procurer des renseignements à l'employeur et au bureau/ service de santé afin de les sensibiliser aux défis du travail par quarts et de faciliter l'adaptation à ce travail. Les objectifs de l'atelier sont de favoriser une compréhension des adaptations biologiques et sociales reliées au travail par quarts; de déterminer et comprendre de quelle façon le travail par quarts touche son mode de vie et sa santé; de trouver et de partager des solutions pour développer sa capacité d'adaptation afin d'améliorer son mode de vie et sa santé ainsi que son milieu 
de travail. Les participants reçoivent un guide à partager avec leur famille et leurs amis. Les ateliers s'adressent à plusieurs groupes, notamment les employés de quartsqui veulent recevoir des conseilssanté pour s'adapter au travail par quarts, les travailleurs ou les travailleuses ainsi que la direction ayant un régime de travail habituel qui désirent accroître leurs connaissances des questions de santé touchant leurs collègues travaillant par quarts, ainsi que les nouveaux employés qui devraient être sensibilisés aux effets du travail par quarts peu après leur entrée en fonction afin de faciliter leur adaptation. I déalement, les membres des familles sont invités à participer. Comme il n'est pas toujours possible de les impliquer, une solution de rechange consiste à encourager les participants et les participantes à partager le $G$ uide de l'employé avec les membres de leur famille et leurs amis.

\section{L'établissement d'une banque de données}

Au cours des ateliers, les participants et les participantes sont invités à compléter un bref questionnaire au sujet des effets du travail par quarts sur leur santé. Ainsi, en 1994, des renseignements ont été obtenus de plus de 900 employés du nord- est de l'O ntario.

Les résultats apportent de nouveaux points intéressants et appuient aussi la documentation existante. $D$ 'après les résultats obtenus, les travailleurs et travailleuses de quarts du nord le l'O ntario

- semblent avoir plus de difficulté à rattraper les heures de sommeil perdu, à s'endormir et à rester endormis.

- croient ne pas avoir assez de temps à consacrer à leur famille et signalent un manque d'occasions de participer aux activités familiales et sociales.

- semblent souffrir de troubles digestifs plus souvent que les personnes ayant un horaire habituel.

- ont tendance à ne pas déjeuner ou à manquer le premier repas après le réveil et sont plus portés à boire du café/ thé ou autres 
boissons caféinées pendant les quatre dernières heures de leur quart ou les quatre heures avant d'aller au lit.

- ont un poids plus élevé que leurscollègues des quarts de jour, tel que mesuré par l'indice de masse corporelle.

II est important de noter que même si des données pertinentes ont été obtenues par ce sondage, certaines limites s'imposent en raison du petit groupe témoin de personnes ayant un régime de travail régulier, du petit nombre de femmes dans l'échantillon, de l'échantillon non-représentatif d'industries, $82 \%$ des répondantes provenant de l'industrie minière et finalement du manque de contrôle des variables confondantes.

Suite à cette première cueillette de données, le questionnaire d'évaluation personnelle a été révisé, en tenant compte des commentaires des participants et des participantes et des professionnels. Ce questionnaire révisé a subi un prétest auprès de trente travailleurs et travailleuses de quarts de diverssecteurs et fait maintenant partie de la trousse d'animation. $\mathrm{N}$ ous espérons continuer de recevoir les renseignements de tous ceux et cellesqui participeront au programme afin de dresser un meilleur profil des travailleurs de quarts et de mieux comprendre et résoudre cette problématique.

L'épidémiologiste du service de santé de Sudbury entreprend également une analyse des données de l'Enquête sur la santé en O ntario 1990 dans le but d'explorer les différences de certaines variables de la santé entre les travailleurs et travailleuses de quarts et ceux et celles ayant un horaire habituel. Les données préliminaires ont aussi été ajoutées à notre guide d'animation.

\section{L'établissement de partenariats}

La promotion de la santé au travail nécessite la collaboration entre les employés, les employeurs, les personnes-ressources et les intervenants et les intervenantes. II est essentiel d'identifier tous les partenaires dans le milieu de travail tels que les syndicats, la 
«L a promotion de la santé au travail nécessite la collaboration entre les employés, les employeurs, les personnes-ressources et les intervenants et les intervenantes. II est essentiel d'identifier tous les partenaires dans le milieu de travail... » direction, le personnel de la santé et de la sécurité du travail. La deuxième section du guide d'animation insiste sur le besoin d'évaluer la culture de l'organisation et suggère des stratégies pour encourager des partenariats efficaces.

Les partenaires les plus importants demeurent les travailleurs de quarts.Wedderburn (1993:239) rappelle l'importance de valoriser l'expérience quotidienne des travailleurs et des travailleuses qui ont développé une sagesse et une expertise dans la façon de gérer leur vie face au travail par quarts. L'approche éducative préconisée doit créer un climat favorisant le partage et intégrant les principes andragogiques. C ette approche axée sur la participation des individus est élaborée à l'intérieur du guide d'animation, tout au long du programme.

À date, le programme facilite surtout une meilleure compréhension du dossier ainsi que le soutien pour l'adaptation au travail par quarts. C'est un point de départ. Les ateliers et les ressources développées permettent une sensibilisation à cette question auprès des employés, des contremaîtres et de la direction. U ne meilleure compréhension stimule des échanges initiaux qui permettront de passer à l'action et éventuellement d'influencer les politiques du milieu de travail ainsi que de faire reconnaître au sein de la communauté, les besoins de la population du travail par quarts. Avec le suivi du programme, il est à souhaiter qu'il sera possible de poursuivre ces buts à long terme.

\section{Évaluation}

En plus d'examiner le processus du développement du projet, le plan d'évaluation cherche à déterminer dansquelle mesure le programme répond aux questions suivantes, découlant des objectifs visés:

- Les ressources développées sont- elles pertinentes, compréhensives et rencontrent-elles les attentes des participants et des participantes? 
- N os connaissances au niveau du lien entre le travail par quarts et la santé ont-elles augmenté?

- Le développement et de la mise en oeuvre du programme ont-ils mené à l'établissement de partenariat?

- Quelles leçons pouvons-nous tirer du développement d'un tel programme?

\section{Les ressources}

Les ressources éducationnelles ont été développées et révisées maintesfois en fonction du feed-badk destravailleuses et travailleurs de quarts. Le guide de l'employé a subi sept révisions majeures pendant la période pilote, suite aux commentaires obtenus après chaque atelier. Pendant la première année, 704 travailleuses et travailleurs ont participé aux ateliers et ont évalué les ressources. Trois groupes foaus différents, s'adressant à des mineurs, des ambulanciers et des infirmières, ont contribué de façon plus détaillée à l'évaluation des ressources et stratégies proposées.

Le contenu du guide des employés a aussi été revu par divers professionnels. Dans le cadre de l'évaluation continue, d'autres données pertinentes ont été obtenues par l'entremise d'entrevues détaillées avec des travailleurs et des travailleuses de quarts, de consultations avec des personnes-clés telles que les infirmières en santé au travail et deux médecins spécial istes du rythme circadien et des experts en al phabétisation. Les comptes rendus des ateliers ainsi que les observations, les notes et les réflexions de l'équipe impliquée dans le projet ont aussi été des sources utiles.

Le guide final de l'employé, le questionnaire d'auto-évaluation, le contenu du guide d'animation ainsi que le format pour les ateliers sont le résultat de ces premières évaluations et incorporent les données préliminaires des participants et participantes au projet, la réaction et les commentaires fournis par les groupes de discussions, le résultat de la revue de la documentation et les consultations avec les experts.

Les évaluations formelles et informelles des ressources et des ateliers sont très positives en ce qui à trait a la pertinence, au contenu et au format. $\mathrm{O}$ a suggéré, pour améliorer le programme, 
des sessions additionnelles ou des sessions plus longues. O n a inclus dans les guides des outils d'évaluation qui ont été développés pour obtenir les commentaires des animateurs ou des animatrices et des participants et participantes au sujet des ressources et des ateliers. Le processus d'évaluation se poursuit grâce au questionnaire d'évaluation qui est inclus dans les guides desanimatrices et animateurs. Ces guides sont présentement en voie de distribution.

Les demandes pour ce programme offrent un autre bon indice de sa valeur et de ses bénéfices. C elles ci se chiffrent à près de cinquante et arrivent non seulement de toutes les parties de la province mais de l'ensemble du C anada. Ces demandes nous arrivent sans promotion du programme; elles sont en grande partie le résultat d'une publicité de bouche à oreille. $U \mathrm{n}$ autre indicateur particulièrement important relié au programme est la demande de consultation de certaines compagnies pour la révision de leurs politiques et de leur horaire du travail par quarts.

Les ressources disponibles ne nous permettaient pas de mesurer l'impact à long terme du programme, tel que les effets sur les congés de maladie, sur les politiques de la compagnie ou sur les connaissances, attitudes et croyances des travailleuses et travailleurs et de leurs familles. C ette composante de l'évaluation fait partie de nos plans pour l'avenir.

\section{Les banques de données}

Lors de l'élaboration du projet, une compilation des articles et des ressources disponibles fut complétée et incorporée au guide d'animation. U ne banque de données sur les effets du travail par quarts a été établie; la cueillette des données et leur analyse se poursuivent. D es champs de recherche pour l'avenir, tels que les effets du travail par quarts sur les travailleuses et sur les enfants, ont déjà été identifiés.

\section{Les partenariats}

Le travail d'équipe nécessité par le développement et la mise en oeuvre du programme a contribué à l'établissement de plusieurs 
nouveaux partenariats. Les procès-verbaux détaillés des réunions, des téléconférences ainsi que les plans d'actions mensuelles nous ont permis de documenter ces résultats.

C es partenariats retrouvent trois groupes d'acteurs provenant du milieu de travail, de l'équipe du projet et des réseaux régional et provincial. À date, des partenariats ont été initiés ou établis au sein de 21 industries dans le $\mathrm{N}$ ord-est de I' $\mathrm{O}$ ntario et regroupent les membres des syndicats, de la direction, du personnel de la santé et de la sécurité du travail dans une collaboration avec les employés des services de santé publique. D es leçons importantes, comme l'importance de bien connaître la culture et la politique du milieu du travail se dégagent du projet. Ces résultats ont été discutés ailleurs (Taillefer, 1994).

Le processus de développement du programme nous a aussi beaucoup appris sur les défis que représente un projet interdisciplinaire à sites multiples. À titre d'exemples, citons les complexités de communication et de coordination, les exigences imprévues de temps (en partie dues à l'enthousiasme et à la demande inattendue pour ce genre de ressources et aux réalités des distances dans le $\mathrm{N}$ ord de la province), la gestion du budget partagée entre deux agences et ainsi que les retards et les coûts reliésà un projet bilingue. U ne évaluation continue du processus a permis d'assurer une communication claire et l'établissement de lignes directrices a facilité le travail tout au long du projet.

\section{Orientations futures}

Le développement du programme illustre l'influence d'un modèle de la promotion de la santé bien connu dans le milieu de la santé publique (ministère de la Santé de l'O ntario, 1991:15). Les plans de promotion de la santé doivent tenir compte des facteurs affectant la santé et font appel aux trois approches qui cherchent à cerner ces problèmes: éducation, politique et soutien communautaire. 
«... afin de promouvoir la santé des travailleurs et des travailleuses de quarts, des changements sont essentiels à quatre niveaux: I'individu, la famille et les amis, le milieu de travail, et la communauté.»
II n'existe pas de réponses ou de stratégies générales, puisque, comme nous rappelle Wedderburn (1990:240), les facteurs personnels, sociaux et organisationnels sont tellement diversifiés. $\mathrm{N}$ éanmoins, afin de promouvoir la santé des travailleurs et des travailleuses de quarts, des changements sont essentiels à quatre niveaux: I'individu, la famille et les amis, le milieu de travail, et la communauté. M entionnons quelques pistes pour l'avenir s'adressant à chacun de ces niveaux du programme. S'il reconnaît les adaptations biologiques et sociales liées au travail par quarts, le travailleur est plus apte à trouver des solutions pour améliorer son mode de vie et sa santé. Les plans sont donc de promouvoir le programme à travers la province afin d'offrir de l'information au plus grand nombre possible d'employés. $U$ ne autre des directions du projet consiste à impliquer plus de collègues, de familles et d'amis afin de renforcer les réseaux de soutien social pour les travailleurs de quarts. II est reconnu qu'un déterminant important de la santé est le réseau social. C ertains experts soulignent que les relations sociales exercent sur la santé un effet aussi important que les facteurs de risque bien connus comme le tabac, I'activité physique, l'obésité ou I'hypertension artérielle (Santé Canada, 1994:17). D ans la même veine, près de la moitié des Canadiens sont d'avis que le fait de passer plus de temps avec leurs familles ou leurs amis intimes les aiderait à améliorer leur santé et leur bien-être (Enquête Promotion de la santé Canada: 1990, 1993:70).

Les plans visent donc à accorder plus d'importance à une approche familiale pour promouvoir un dialogue entre le travailleur ou la travailleuse et les membres de sa famille. Cet échange permettrait à la famille d'identifier les stratégies propres à sa situation. Pour mieux comprendre l'impact du travail par quarts sur la famille, de plus amples recherches sont nécessaires. $N$ os données suggèrent que nous devrions en fait parler non seulement du travailleur ou de la travailleuse de quarts mais de la famille de quarts.

Suite aux activités éducatives qui ont créé une sensibilisation au dossier dans le milieu du travail, la prochaine étape du programme, à ce niveau, serait d'appuyer les efforts qui visent à influencer les politiques du milieu de travail. Par exemple, la 
direction devrait prendre en considération le fait qu'un horaire de rotation vers l'avant, du jour au soir, est généralement préférable à celui en sens inverse. II est possible que les rotations moins fréquentes facilitent aussi l'adaptation au travail par quarts.

$D$ 'autre éléments doivent également être reconnus, surtout durant le quart de nuit, notamment, l'accès à l'alimentation, l'importance d'une bonne illumination et une assignation judicieuse des tâches pendant la nuit. La revue Santé et séaurité au travail offre un résumé des aspects à améliorer dans le lieu de travail (1990:2).

La conception des horaires doit répondre aux exigences de production mais doit aussi tenir compte des effets du travail par quarts sur la santé, la sécurité et la prévention des accidents industriels. Si possible, il est bon pour les employeurs de prendre en considération certaines caractéristiques de leur main d'oeuvre telles que l'âge et même les besoins particuliers des parents avec des enfants de moins d'un an, des familles monoparentales et des femmes enceintes.

U ne campagne pour sensibiliser la communauté au nombre de travailleurs de quarts et à leurs besoins particuliers serait une autre stratégie pour renforcer le programme. La communauté peut jouer un rôle dans l'adaptation des travailleurs et des travailleuses de quarts en essayant de répondre aux besoins de ce secteur souvent oublié. U n exemple serait une meilleure disponibilité des services communautaires tels que les dépanneurs, les épiceries et autres magasins, les services de santé et services sociaux, les cours éducatifs, les bibliothèques, les cinémas et les centres de conditionnement physique.

\section{Conclusion}

Les compagnies peuvent choisir trois options pour la mise en oeuvre du programme. Elles peuvent recevoir une copie du guide d'animation afin d'animer leur propre atelier pour leursemployés; inviter l'infirmier ou l'infirmière hygiéniste ou la nutritionniste 
du service de santé local à offrir une session de formation sur I'utilisation du guide, à l'équipe de santé et de sécurité au travail; ou finalement, inviter l'infirmier ou l'infirmière hygiéniste ou la nutritionniste du service de santé local à offrir l'atelier aux employés.

L'option appropriée dépend de la taille et de la structure de la compagnie ainsi que de l'engagement de la direction. U n effort conjoint de la direction et des employés serait l'idéal. Le comité de santé et de sécurité peut parfois jouer un rôle-clé car ces comités représentent les travailleurs et les travailleuses, le syndicat et la direction de l'entreprise. L'organisation et la mise en oeuvre des ateliers pourraient créer une sensibilisation initiale et susciter par la suite un effort collectif pour améliorer les conditions du travail par quarts.

Dans la mesure du possible, les services de santé publique préfèrent jouer un rôle consultatif en offrant les outils nécessaires et en créant des multiplicateurs dans le milieu de travail afin d'assurer la propagation maximale des ateliers. Cette approche consultative et formative assure le maintien du programme. En ce qui concerne le suivi, les intervenants et intervenantes cherchent à mousser et à appuyer l'action collective qui apportera les changements désirés dans le milieu du travail.

Pour ceux et celles qui désirent entreprendre un programme dans le milieu du travail pour les travailleurs et travailleuses de quarts, la démarche proposée par $\mathrm{M}$ artin et Paiement est appropriée (1990:7). C es auteurs nous proposent cinq étapes afin de développer des conditions de travail favorables à la santé des travailleurs et des travailleuses, soit l'analyse du milieu, l'établissement d'un consensus, la planification opérationnelle du mandat, la réalisation des activités, l'évaluation et le suivi. C ette approche est reflétée dans notre programme et est résumée dans le guide d'animation avec un accent particulier sur l'importance du marketing du programme.

La question de santé et sécurité au travail est devenue prioritaire pour les compagnies, les syndicats et les employés. $D$ ans le $R$ apport du $G$ roupe consultatif sur le temps de travail et la répartition $d u$ 
Reflets

travail (D éveloppement des ressources humaines C anada, 1994: 71), les employeurs, les syndicats et les employés sont invités à accorder encore plusd'importance aux questions relativesau temps de travail. II existe aujourd'hui, une meilleure compréhension des effets du travail de quarts, un plus grand nombre de personnes impliquées et une meilleure appréciation du lien qui existe entre la santé, la sécurité et la production. II est donc essentiel de faciliter l'adaptation à ce mode de vie pour ceux et celles qui travaillent par quarts et pour leurs familles. Les bienfaits sont multiples: une meilleure qualité de vie professionnelle, personnelle et sociale.

Les auteures désirent remercier les autres membres de l'équipe du travail par quarts: Judy H uggins, V ic Sahai, G wen W ilson. U n merd spédal à Suz anne P elletier, Paulette Picard et Joelle Z orz etto pour leur assistance dans la préparation du manuscrit.

\section{Bibliographie}

BAR ET TE, Lise (1994). W orkplace H ealth Promotion Survey, Sudbury, Service de santé publique de Sudbury.

CALDW ELL, J. Paul (1995). Sleep,Toronto, Key Porter Books.

COLLIGAN, M ichael et Roger ROSA (1990). «Shiftwork Effects on social and family life», 0 coupational M edicine, vol. 5, no 2, avril-juin, 315-322.

COLQU OUN,W.P. et J. RUTENFRANZ (1980). «ntroduction», dans W.P. Colquhoun et J. $R$ utenfranz ed. Studies in Shiftwork, London, Taylor \& Francis, ix-xi.

COMMISSION DE L'HYGIENE ET DE LA SÉCURITÉ AU TR AVAIL DU NOUVEAU BRU N SW ICK (1989). «e travail par quart nuit au rendement humain», Santé et sécurité au travail, Frédéricton, été, 2.

DÉVELO PPEM EN T DES R ESSO U R CES HU M AIN ES CAN ADA (1994). R apport du G roupe consultatif sur le temps du travail et la répartition du travail, $\mathrm{O}$ ttawa, $\mathrm{M}$ inistre des Approvisionnements et Services $C$ anada.

D OT TO, L.(1990). A sleep in the fast lane, Toronto, Stoddart.

KN U TSSO N, A., B. JO N SSO N,T. AKER STEDT et K.ORTH-GOMER (1986). «ncreased risk of ischaemic heart disease in shift workers», $T$ he $L$ ancet, 89-91.

LEN N ER N AS, M ., L. H AM BR AEU S et T.AKER STEDT (1993). «N utrition and shiftwork: the use of meal classification as a new tool for qualitative/ quantitative evaluation of dietary intake in shiftworkers», E rgonomics, vol. 36, no 1-3, 247-254. 
MINISTÈR E DE LA SANTÉ DE L'ONTARIO (1992). L'enquête sur la santé en 0 ntario 1990, Toronto.

M IN ISTĖR E DE LA SAN TÉ DE L'ONTARIO (1991). G uide de planification de la promotion de la santé communautaire, Toronto.

M O N K, Timothy et Simon FO LKAR D (1992). M aking Shiftwork Tolerable, Washington, Taylor \& Francis.

M O ORE-EDE, M. (1993). «C ircadian physiology expert offers nine ways to keep you alert», $C$ anadian 0 caupational Safety, 3.

PAIEM EN T, M arguerite et C atherine M ARTIN, (1990) L'intervention éducative pour la promotion de la santé au travail, C ollection Santé - Société, Q uébec, M inistère de la santé et des services sociaux

SAN TÉ CAN ADA (1994). Stratégies d'amélioration de la santé de la population, 0 ttawa, M inistre des A pprovisionnements et Services $C$ anada.

SAN TÉ ET BIEN -ÊTR E SOCIAL CAN ADA (1992). Le travail par équipes, 0 ttawa, M inistre des A pprovisionnements et Services $C$ anada.

SANTÉ ET BIEN -ÊTRE SO CIAL CAN ADA (1993). Enquête canadienne. Promotion de la santé $C$ anada 1990: $R$ apport technique, $O$ ttawa, $M$ inistre des A pprovisionnements et Services $C$ anada.

SM O LEN SK Y, M .H. (1980). «T he chronoepidemiology of occupational health and shiftwork», dans A. R einberg, N.V ieux et P. A ndlauer, éd. vol. 31, N ight and Shiftwork B iological and Social A spects, N ew York, Pergamon Press, 51-67.

TAILLEFER , T. (1994). «es personnes qui travaillent par quarts et les rapports de pouvoir sains.» Conférence annuelle de l'Association pour la santé publique de I'O ntario,Toronto.

W ED D ER BU R N ,A lexander (1993). «Teaching grandmothers to suck eggs: D o shiftworkers need rules or guidelines?», E rgonomics, vol. 36, no 1-3, 239-246. 